\title{
Closed form solution for radial distortion estimation from a single vanishing point ${ }^{1}$
}

\author{
Horst Wildenauer \\ horst.wildenauer@gmail.com \\ Branislav Micusik \\ branislav.micusik@aic.ac.at
}

\author{
Vienna University of Technology \\ Vienna, Austria \\ AIT Austrian Institute of Technology \\ Vienna, Austria
}

\begin{abstract}
We focus on the estimation of radial lens distortion utilizing vanishing points detected in single images. Recent methods for vanishing point detection either ignore radial distortion completely, or assume only weak distortion, which is accounted for after vanishing point estimation. Unfortunately, if strong radial distortion is present the effects are detrimental to the extraction of vanishing points and such algorithms are bound to fail. To overcome this limitation, we suggest a closed-form solution for the problem of simultaneously estimating a single vanishing point and radial distortion from three distorted image lines. By utilizing our solver in a RANSAC-like algorithm, we arrive at a unified camera calibration approach, which in addition to stably estimating radial distortion, computes the camera's focal length if at least two orthogonal vanishing points are present. Based on extensive experiments we show that our approach presents a significant contribution to the state-of-the-art in camera self-calibration from single images.
\end{abstract}

\section{Introduction}

Radial lens distortion found in real, most notably (cheap) off-the-shelf medium to wide angle optics can be quite severe. This complicates the analysis of digital imagery, as deviations from the pin-hole camera model are significant. A-priori calibration of lens distortion, possibly alongside the camera's other parameters [18], remedies the problem. However, such techniques are not applicable to zoom lenses and more importantly, require access to the camera. Other approaches make use of correspondences in multiple views of a moving camera, e.g. by utilizing constraints from epipolar geometry $[2,9,12]$. These methods require sufficiently overlapping views and suffer from the lack of texture commonly encountered in indoor environments.

Another class of algorithms focuses on estimating lens distortion from single views, e.g. by imposing the constraint that straight scene lines have to appear straight in the image $[7,8,14]$. These methods depend on extraction of long, smoothly curved edges and get thrown off easily if a portion of such curves originates from non-linear scene structures. For this problem, the use of vanishing points provides a distinct advantage: When a significant number of image edges groups w.r.t. to a common vanishing point, it is far more likely that they stem from parallel scene lines, than being raised by some arbitrary curved

\footnotetext{
${ }^{1}$ This research has been supported by funding from the Austrian Research Promotion Agency (FFG) project LOLOG 3579656 and PAMON 835916. 

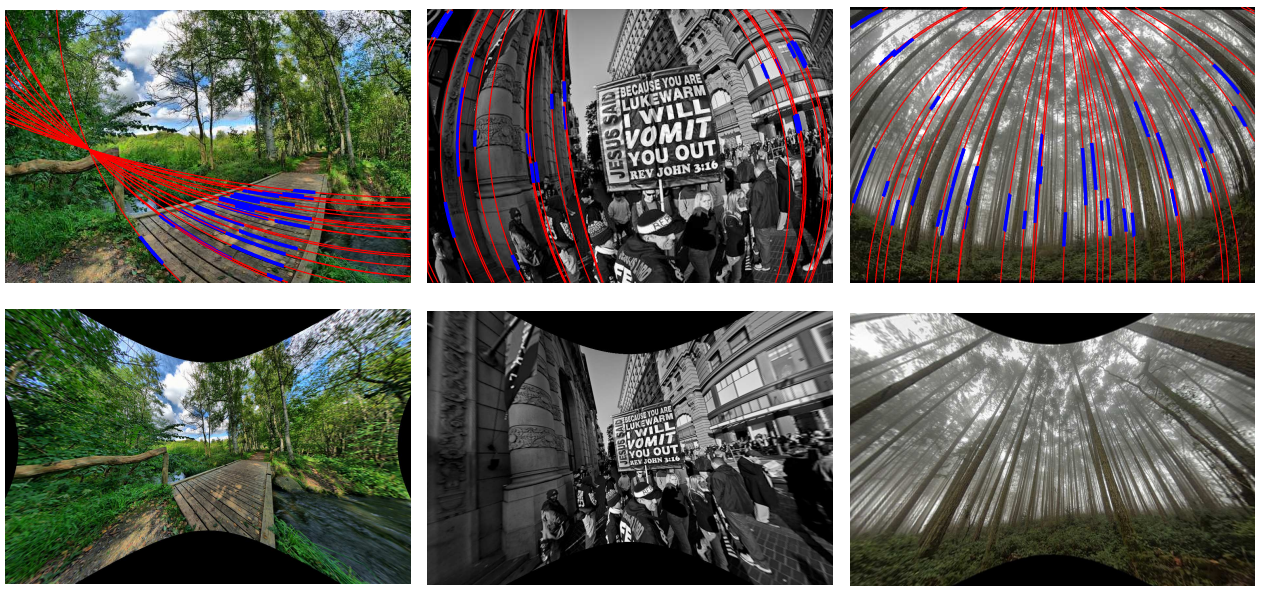

Figure 1: Exemplary results: Circular arcs (blue) grouped w.r.t. to a vanishing point, indicated by converging circles (first row). Automatically undistorted images. (second row).

structures. For weakly distorted images this has been considered by Bräuer-Burchardt and Voss [4]. Similarly, in [10] Grammatikopoulos et al. attempt to account for weak radial distortion in the context of camera calibration from orthogonal vanishing points. They propose re-detection of straight lines together with a refinement of focal length, radial distortion, and vanishing points starting from initial vanishing point estimates obtained by a standard linebased approach. Hughes et al. [11] utilize vanishing points to estimate the strong distortion effects present in fish-eye lenses. However, their method is designed for calibration patterns.

\subsection{Contributions}

We concern ourselves with the detection of vanishing points and concurrent estimation of radial distortion, using sets of edge segments extracted from a single view. This has been attempted before [4, 10], but the proposed algorithms share the same deficiencies: a) They assume weak radial distortion such that a first guess at vanishing point positions can be made without accounting for distortion. b) When dealing with radial distortion effects in subsequent refinement stages, the error is quantified in the undistorted image. This creates a significant bias towards distortion estimates shrinking the undistorted image $[8,15]$.

We address both limitations utilizing a simple RANSAC strategy for grouping distorted line segments w.r.t. to vanishing points. The main contribution lies in the generation of RANSAC hypotheses: We suggest a closed form solution for jointly estimating radial distortion and the vanishing point position from a set of three distorted image edges. Furthermore, the method avoids bias by assessing the consistency of an edge segment w.r.t. a vanishing point directly in the distorted image. See Fig. 1 for results obtained with our algorithm.

As an extension of the one vanishing point case, we adopt our solution in a more general RANSAC-based framework, which in addition to estimating radial distortion from three segments, adds two more segments to compute the focal length by identifying up to three orthogonal vanishing points. In this approach, the assumption of orthogonality is necessary for focal length computation, but does not affect the estimation of radial distortion. To summarize, our contributions are: 
- A closed-form solution for estimating a vanishing point and radial distortion from three distorted image lines together with an efficient consistency measure for grouping sets of distorted lines w.r.t. their vanishing points.

- The adoption of our solver in a unified and robust camera self-calibration framework based on RANSAC, which computes radial distortion and focal length, as well as three orthogonal vanishing points, from a total of five segments.

- Extensive experiments show that the approach is computationally efficient and can handle significantly distorted images as well as images unaffected by radial distortion.

\section{Lens distortion model}

For our work, we adopt the so-called division model [3] made popular by Fitzgibbon [9]. In this model the mapping from a distorted image point $\mathbf{x}$ to the undistorted point $\tilde{\mathbf{x}}$ is given by

$$
C: \quad \tilde{\mathbf{x}}=\mathbf{x} /\left(1+\lambda r^{2}\right) .
$$

Here, $r=\sqrt{x^{2}+y^{2}}$ is the distance of the distorted point to the distortion center (the origin of the coordinate frame), which is typically set to the center of the image $[9,16]$. The coefficient $\lambda$ controls the degree of distortion: At $\lambda=0$ no distortion is present, while $\lambda<0$ and $\lambda>0$ account for barrelling and pincushion distortion respectively. For the rest of the paper, we assume normalized image coordinates, such that $0 \leq r<1$.

Intriguingly, it can be shown [2] that under the division model a straight line $\tilde{\mathbf{I}}=\left(\tilde{l}_{1}, \tilde{l}_{2}, \tilde{l}_{3}\right)^{\top}$ is distorted into a circle with center $\left(x_{c}, y_{c}\right)$ and radius $R$ given by

$$
x_{c}=-\frac{\tilde{l}_{1}}{2 \lambda \tilde{l}_{3}}, \quad y_{c}=-\frac{\tilde{l}_{2}}{2 \lambda \tilde{l}_{3}}, \quad R=\sqrt{\frac{l_{1}^{2}+l_{2}^{2}}{4 \lambda^{2} l_{3}^{2}}-\frac{1}{\lambda}}=\sqrt{x_{c}^{2}+y_{c}^{2}-\frac{1}{\lambda}} .
$$

The circle degenerates to a line for either $\lambda=0$ or $\tilde{l}_{3}=0$, i.e. 1 passing through the origin.

In [15], Strand and Hayman utilize this observation to correct radial distortion. They demonstrate that superior results compared to methods minimizing the error in the undistorted image [7] can be achieved by fitting circles to image edges in the distorted image. Basically, once a circle has been identified in an image, the rightmost term in (2) can be used to compute $\lambda$ from the circle's midpoint and radius. Note however, that this approach critically depends on long, sufficiently curved edges which may only be found close to image boundaries. When circles are fit to short and therefore almost straight edges, the estimated parameters are are known to be unstable [5].

\section{Estimating radial distortion from circular arcs}

In spirit of Strand and Hayman [15], we will utilize circular arcs extracted from edges as basic feature for our approach. A short account of the adopted technique is given in 3.1. However, while such features are plentiful in images of man-made environments, one cannot rely on sufficient length or curvedness for immediate estimation of distortion. In Sec. 3.2 we will show that improved results can be achieved by utilizing the assumption of a common vanishing point. We formulate the simultaneous computation of a vanishing point and 

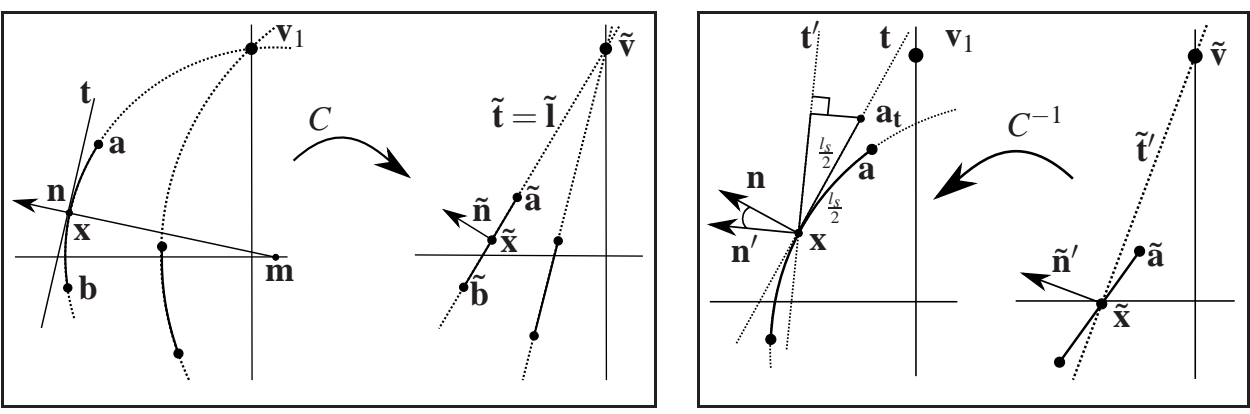

Figure 2: Left: The tangent $\mathbf{t}$ to the circular arc (circle with midpoint $\mathbf{m}$ ) transformed into the undistorted image coincides with the original line Ĩ passing through the vanishing point (Sec. 3.2). Right: The consistency of a circular arc w.r.t. to a vanishing point is assessed by constructing the ideal line $\tilde{\mathbf{t}}^{\prime}$ in the undistorted image and warping it to $\mathbf{t}^{\prime}$ in the distorted one. The error is the perpendicular distance from point $\mathbf{a}_{\mathbf{t}}$ on line $\mathbf{t}$ to $\mathbf{t}^{\prime}$, see Sec. 3.3 for details.

radial distortion as the estimation of a pencil of circle tangents transformed into the undistorted image. The main trick is that we under-parametrize the circles such that enforcing the constraint of a common vanishing point turns the formulation into a simple generalized eigenvalue problem. This can be solved in closed form for the minimal number of three circles required by our method.

\subsection{Circular arc detection}

For arc segment detection we employ the Canny detector. The resulting edges are split into circular arcs, similar to typical polygonalization procedures based on the Douglas-Peuker algorithm. Specifically, the procedure recursively splits edges into sub-segments until a circle can be successfully fit, i.e. the maximum deviation of all pixels is smaller than a predefined threshold. For each extracted arc smoothed estimates of the arc's midpoint and the tangent at that point are computed from the fitted circle. In preliminary experiments, we compared the algebraic Taubin circle fit and the fit based on minimizing geometric distance using Levenberg-Marquardt minimization [5]. The later had no noticeable impact on the quality of radial distortion estimation and we selected the more efficient Taubin method.

\subsection{A tangent-based solver}

Suppose we are given a point $\mathbf{x}=(x, y)^{\top}$ on a circular arc together with the normal vector $\mathbf{n}=(u, v)^{\top}$ at that point, obtained with the method from Sec. 3.1. If we undistort $\mathbf{x}$ and map $\mathbf{n}$ accordingly to its undistorted image $\tilde{\mathbf{n}}$, the line $\tilde{\mathbf{t}}$ parametrized by the $\tilde{\mathbf{x}}$ and $\tilde{\mathbf{n}}$ coincides with the line $\tilde{\mathbf{l}}$ generating the circle. See Fig. 2(left) for an illustration of this concept.

From elementary differential geometry we know that for a differentiable transformation of a curve, the normals of the curve are mapped to the normals of the curve's image by the transposed inverse of the Jacobian of that transformation. In our problem $\mathbf{x}$ is transformed into the undistorted point $\tilde{\mathbf{x}}$ by $\mathcal{C}(1)$, for which the Jacobian is

$$
\mathrm{J}_{\mathcal{C}}=\frac{1}{\left(1+\lambda r^{2}\right)^{2}}\left(\begin{array}{cc}
1+\lambda r^{2}-2 \lambda x^{2} & -2 \lambda x y \\
-2 \lambda x y & 1+\lambda r^{2}-2 \lambda y^{2}
\end{array}\right)
$$


Note that $\mathrm{J}_{\mathcal{C}}=\mathrm{J}_{\mathcal{C}}^{\top}$ and we can write the normal vector $\tilde{\mathbf{n}}$ at the undistorted point $\tilde{\mathbf{x}}$ as

$$
\tilde{\mathbf{n}}=J_{\mathcal{C}}^{-1} \mathbf{n}=\frac{1+\lambda r^{2}}{1-\lambda r^{2}}\left(\begin{array}{l}
u+\lambda r^{2} u+2 \lambda v x y-2 \lambda u y^{2} \\
v+\lambda r^{2} v+2 \lambda u x y-2 \lambda v x^{2}
\end{array}\right) .
$$

Using (1) and (4), the undistorted tangent line $\tilde{\mathbf{t}}=\left(\tilde{t}_{1}, \tilde{t}_{2}, \tilde{t}_{3}\right)^{\top}$ passing through $\tilde{\mathbf{x}}$ with normal $\tilde{\mathbf{n}}$ is given by

$$
\tilde{\mathbf{t}}=\left(\begin{array}{c}
\tilde{\mathbf{n}} \\
-\tilde{\mathbf{n}}^{\top} \tilde{\mathbf{x}}
\end{array}\right)=\left(\begin{array}{c}
\tilde{\mathbf{n}} \\
-\tilde{\mathbf{n}}^{\top} \mathbf{x} \frac{1}{1+\lambda r^{2}}
\end{array}\right)=\frac{1+\lambda r^{2}}{1-\lambda r^{2}}\left(\begin{array}{c}
u+\lambda r^{2} u+2 \lambda v x y-2 \lambda u y^{2} \\
v+\lambda r^{2} v+2 \lambda u x y-2 \lambda v x^{2} \\
-u x-v y
\end{array}\right) .
$$

We may drop the common scale factor and substitute $r^{2}=x^{2}+y^{2}$. Then, rearranging of terms gives the desired parametrization of the undistorted tangent line:

$$
\tilde{\mathbf{t}}=\left(\begin{array}{c}
u \\
v \\
-u x-v y
\end{array}\right)+\lambda\left(\begin{array}{c}
u x^{2}+2 v x y-u y^{2} \\
v y^{2}+2 u x y-v x^{2} \\
0
\end{array}\right)
$$

Recall that here the only unknown is $\lambda$. The transformed tangent $\tilde{\mathbf{t}}$ has to pass through the undistorted vanishing point $\tilde{\mathbf{v}}=\left(\tilde{v}_{1}, \tilde{v}_{2}, \tilde{v}_{3}\right)^{\top}$ so that $\tilde{\mathbf{t}}^{\top} \tilde{\mathbf{v}}=0$. Stacking the equations $\tilde{\mathbf{t}}_{i}^{\top} \tilde{\mathbf{v}}_{i}=0, \quad i=1,2,3$ of three tangent lines, we obtain the generalized eigenvalue problem

$$
(\mathrm{D}+\lambda \mathrm{E}) \tilde{\mathbf{v}}=\left(\left[\begin{array}{lll}
d_{1} & d_{2} & d_{3} \\
d_{4} & d_{5} & d_{6} \\
d_{7} & d_{8} & d_{9}
\end{array}\right]+\lambda\left[\begin{array}{lll}
e_{1} & e_{2} & 0 \\
e_{3} & e_{4} & 0 \\
e_{5} & e_{6} & 0
\end{array}\right]\right) \tilde{\mathbf{v}}=\mathbf{0}
$$

where $D, E$ are the so called design matrices. Letting $\operatorname{det}(\mathrm{D}+\lambda \mathrm{E})=0$ and rewriting, it can be shown that the characteristic polynomial is a simple quadratic of the form

$$
\lambda^{2} c_{2}+\lambda c_{1}+c_{0}=0 .
$$

Solving for $\lambda$ gives at most two real roots $\lambda_{1,2}$. Due to the underparametrization of the involved circles both solutions are geometrically correct, representing two different triplets of circles meeting in a common vanishing point in the undistorted image. Once $\lambda$ is computed, we plug it back into (6) to obtain the three undistorted lines from which intersecting any two gives the vanishing point.

At this point, it is convenient to discuss the cases when our solver fails to give a solution. We identified three critical cases, which can be easily derived from equations (4) and (6):

- Three tangent lines passing through the distortion center: For any line passing through the distortion center, the orientation of the normal $\tilde{\mathbf{n}}$ is not affected by changing $\lambda$. Consequently, the tangent lines are not altered and the distortion cannot be estimated.

- Three parallel tangent lines with normals (at the point of tangency $\mathbf{x}$ ) pointing towards the distortion center: Again, the normal vectors are only scaled by radial distortion, such that the tangent lines will only be shifted, but stay parallel for changing $\lambda$.

- Three tangent lines lie on the same circle: In that case $\lambda$ is still computable as the trivial solution making $\tilde{\mathbf{v}}=\mathbf{0}$, i.e. the $\lambda$ where all three undistorted tangent lines are equivalent up to scale, such that the intersection point is undefined. 

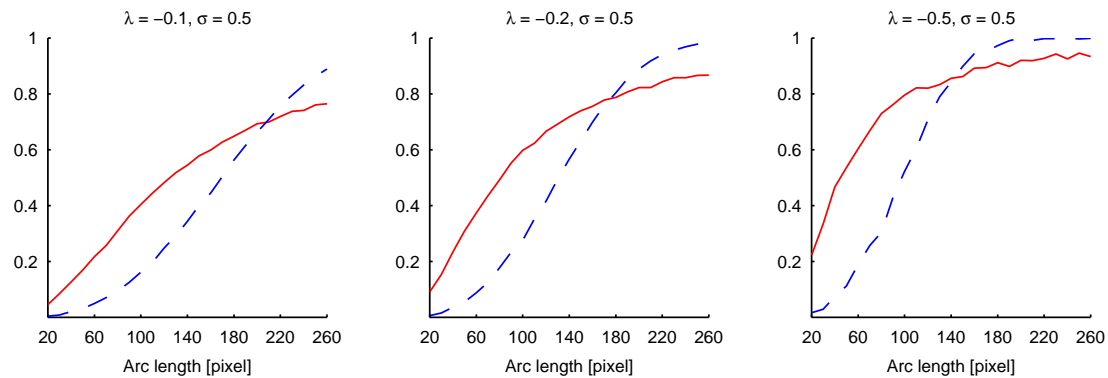

Figure 3: Synthetic data results: The ordinate shows the fraction of successful trials (relative error in $\lambda$ smaller than 5\%) versus length of circular arcs on the abscissa. The solid red line denotes our method, the dashed blue line stands for the best-of-three circle fit.

\subsection{Assessing the consistency of circular arcs}

In this section we will consider a method to measure the consistency of arc segments w.r.t. a vanishing point $\tilde{\mathbf{v}}$ for a given $\lambda$. To avoid bias in distortion estimation (Sec. 1.1) the error will be measured in the original image, as this is where the uncertainty originates. Specifically, we asses the error utilizing the same representation as for computing the vanishing point. For the arc midpoint $\mathbf{x}$ its undistorted image $\tilde{\mathbf{x}}$ is obtained by (1). Then we compute the normal $\tilde{\mathbf{n}}^{\prime}$ of the ideal line $\tilde{\mathbf{t}}^{\prime}$ connecting $\tilde{\mathbf{v}}$ and $\tilde{\mathbf{x}}$, and use relation (4) to find its image

$$
\mathbf{n}^{\prime}=\mathrm{J}_{C} \tilde{\mathbf{n}}^{\prime}=\mathrm{J}_{C}\left(\begin{array}{l}
\tilde{v}_{3} \frac{y}{1+\lambda r^{2}}-\tilde{v}_{2} \\
\tilde{v}_{1}-\tilde{v}_{3} \frac{x}{1+\lambda r^{2}}
\end{array}\right)
$$

in the distorted domain. We define the consistency of a circular $\operatorname{arc} s$ w.r.t. $\tilde{\mathbf{v}}$ as

$$
\operatorname{dist}(s, \tilde{\mathbf{v}}, \lambda)=\frac{l_{s}}{2} \sin \angle\left(\mathbf{n}, \mathbf{n}^{\prime}\right),
$$

where $l_{s}$ is the arc's length in pixels. The weighting factor $l_{s}$ represents a means of allowing less angular deviation of the normals for longer (presumably more accurate) arcs. Another explanation is that (10) is the perpendicular distance of the image of the ideal tangent line $\mathbf{t}^{\prime}$ (with normal $\mathbf{n}^{\prime}$ ) to the point $\mathbf{a}_{\mathbf{t}}$ on $\mathbf{t}$ lying $l_{s} / 2$ pixels away from $\mathbf{x}$, see Fig. 2(right). For $\lambda=0$, this corresponds to the line segment-based consistency employed in [17].

We may note that the proposed measure is not unambiguous. However, this caused no problems during all our tests, suggesting that the accidental alignment of a circle not compatible with $\tilde{\mathbf{v}}$ through the same point $\mathbf{x}$ with identical normal $\mathbf{n}$, is highly unlikely. Besides, a simple but slightly more costly option without the ambiguity is to distort the ideal line $\tilde{\mathbf{t}}^{\prime}$ into a circle passing through $\mathbf{x}$ using (2) and then evaluate the geometric error of two additional points on the arc. In preliminary experiments we compared both options, but encountered no significant differences.

\subsection{Testing the solver on synthetic data}

To get an idea about the behaviour of our minimal solver under noisy conditions, we simulated the output of the circle detector described in Sec. 3.1. In a $640 \times 480$ image circular arcs were created by sampling equally spaced points along circles (compatible with a vanishing point), such that their number approximated the given arc length. Furthermore, the 
point locations were subjected to additive, zero mean Gaussian noise. To each arc, a circle was fit and estimates for the arc's midpoint and associated tangent were extracted.

In a Monte-carlo experiment, we repeatedly (10000 times) created triplets of equal length arcs and computed individual estimates of $\lambda$ from each circle fit (see Sec. 2), as well as by our method. For the three circle fits, the $\lambda$ closest to the ground truth value was retained. Our solver gives up to two solutions per triplet, of which we selected the best $\lambda$. For each trial, the number of successes (relative deviation from ground truth $\lambda$ below $5 \%$ ) was recorded. The experiment was conducted for noise levels in the range of $0.2<\sigma<1$ and arc lengths increasing from 20 to 260 pixels. Due to the lack of space, we only give a representative example of the outcome. Fig. 3 illustrates the results for three values of $\lambda$ at a noise level of $\sigma=0.5$. As can be seen, our method drastically improves the quality of the estimation for short arcs, regardless of the distortion being moderate $(\lambda=-0.1)$, or severe $(\lambda=-0.5)$.

\section{RANSAC}

So far, we have gathered all ingredients for a straight-forward implementation of a RANSACbased method to estimate distortion from a single vanishing point. In Fig. 1 typical results obtained with this algorithm are depicted. As one can see, even images of natural scenes may be used to compute visually pleasing results. Unfortunately, many images have more than one vanishing point and if the dominant one is close to the image center, radial distortion cannot by reliably estimated. The solution to this problem is largely application dependent, and can be attacked by using several vanishing points [4], or by searching for a specific vanishing point, e.g. the vertical one $[1,13]$.

Recently, Wildenauer and Hanbury [17] presented an algorithm for computing three orthogonal vanishing points and the camera's focal length by using sets of four image lines. Their method fixes the principal point in the image center and computes a vanishing point from two lines. Two more vanishing points and focal length are then estimated from the first vanishing point and the remaining lines. This approach can be easily extended to also deal with radial distortion: One needs to do only marginally more than replacing the computation of the first vanishing point with our solver.

In the following, we give the specifics of the resulting RANSAC implementation: A minimal sample set (MSS) of 5 tangents is drawn, of which 3 are taken to compute $\lambda$ and the undistorted vanishing point $\tilde{\mathbf{v}}_{1}$. Then, the 2 remaining tangents are undistorted and fed, together with $\tilde{\mathbf{v}}_{1}$, into the line-based solver [17]. This adds the estimate of the focal length $f$ and two more undistorted vanishing points to the RANSAC hypothesis $\mathcal{H}=\left\{\lambda, f, \tilde{\mathbf{v}}_{1,2,3}\right\}$. Each tangent triplet generates up to 2 solutions for $\lambda$. And, for each $\lambda$ the line-based solver outputs up to 3 focal length estimates. Since there are 10 combinations of drawing 3 tangents from 5 , a maximum of $60=10 \times 2 \times 3$ hypotheses has to be evaluated for each MSS.

In the evaluation step, the cardinality of the consensus set of a hypothesis $\mathcal{H}$ is computed. Here, a circular arc is classified as inlier w.r.t. the hypothesis $\mathcal{H}$, if it has an error $\operatorname{dist}\left(\mathrm{s}, \tilde{\mathbf{v}}_{i}, \lambda\right)$ (see Sec. 3.3) smaller than a predefined threshold $\theta$ for any of the three vanishing points $\tilde{\mathbf{v}}_{1,2,3}$. RANSAC iterates until $k_{\text {data }}$ hypotheses have been evaluated and reports the hypothesis with the largest consensus set.

The advantage of this scheme is two-fold: (a) By using more vanishing points the algorithm covers a larger portion of the data and thereby stabilizes the selection of a vanishing point for radial distortion estimation. (b) If orthogonal vanishing points are present, we get an estimate of the focal length for free. This, however, is not critical for distortion estimation. 


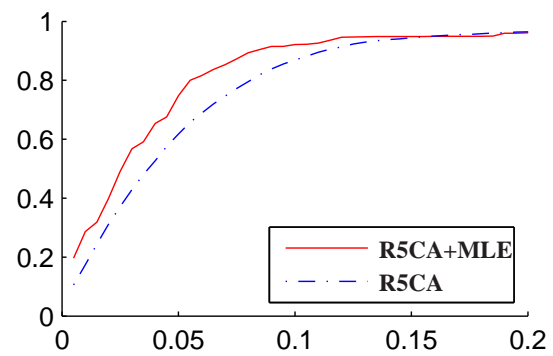

Relative error in distortion coefficient $\lambda$
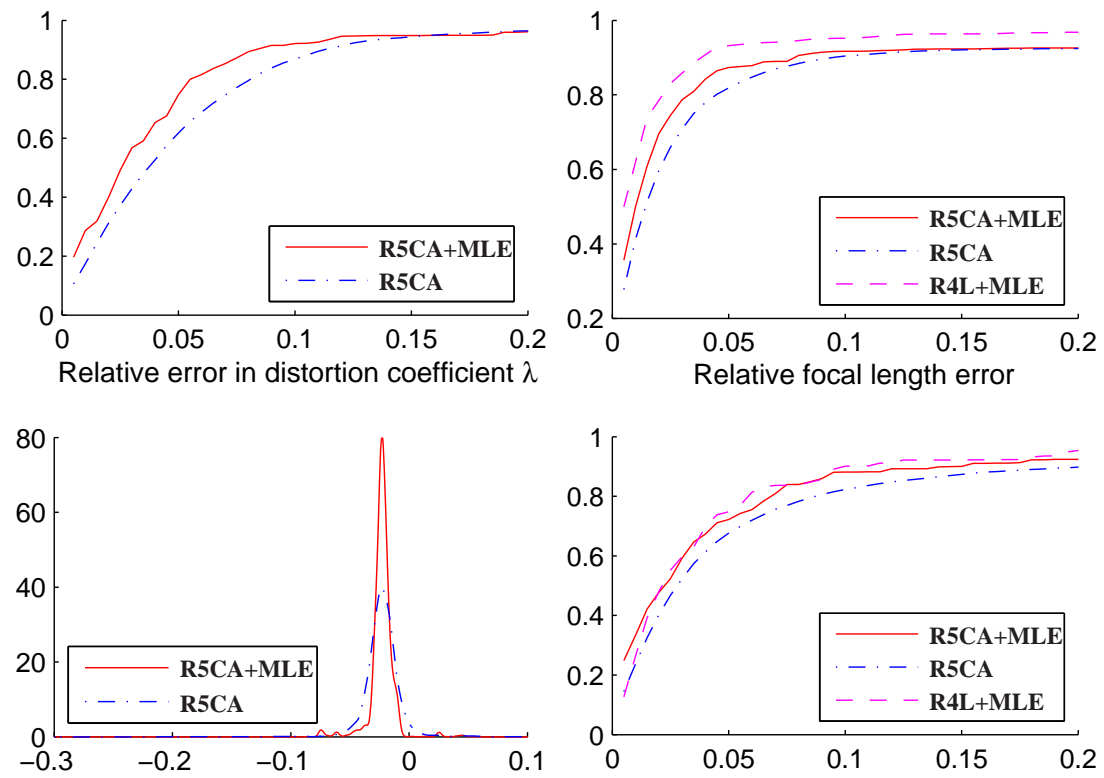

Radial distortion coefficient $\lambda$

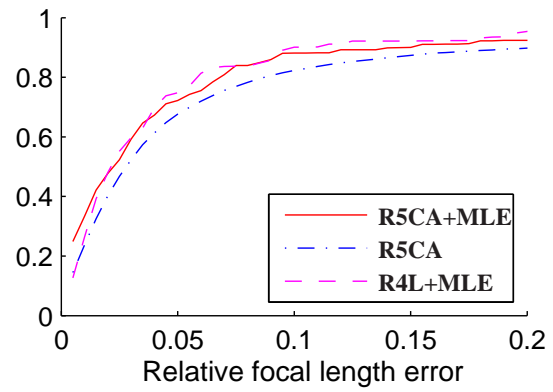

Figure 4: Top row: Cumulative histograms of relative focal length and radial distortion error for our data set. The ordinate shows the fraction of trials with error less than the respective value on the abscissa. Bottom row: Distribution of estimated distortion coefficients (left) and cumulative histogram of relative focal length error for YUD (right).

After the RANSAC stage, we conduct an additional refinement of estimates by simultaneous non-linear optimization of radial distortion, focal length, and vanishing point positions. For this we adopt the Maximum Likelihood Estimator suggested in [17] by simply including the estimation of $\lambda$ and our consistency measure. For the rest of the paper, we denote our RANSAC only implementation as R5CA and the version with refinement as R5CA+MLE.

\section{Experimental results}

For tests on real data we used a Canon EOS 500D mounting a Walimex Pro 8mm fishEye lens with $\approx 170^{\circ}$ horizontal field of view. The acquired images were downscaled to a resolution of $1188 \times 792$ from which a $60 \%$ cutout was retained. We pre-calibrated the camera with distortion center and principal point fixed at the center of the image, resulting in a focal length of $f_{g t}=446$ pixels and radial distortion $\lambda_{g t}=-0.295$.

We acquired a set of 102 images showing mainly street and indoor views of urban environments. For an evaluation of R5CA and R5CA+MLE we assessed the relative error in radial distortion and focal length estimates w.r.t. the ground truth. In addition, we undistorted the images using the ground truth $\lambda_{g t}$ and computed focal length estimates utilizing the vanishing point detector R4L+MLE proposed in [17]. In all tests, the number of iterations was $k_{\text {dat } a}=4000$ and the inlier threshold was set to $\theta=0.5$ pixel. For each image, the algorithms were run 100 times and we report the cumulative results in Fig. 4, top row. 

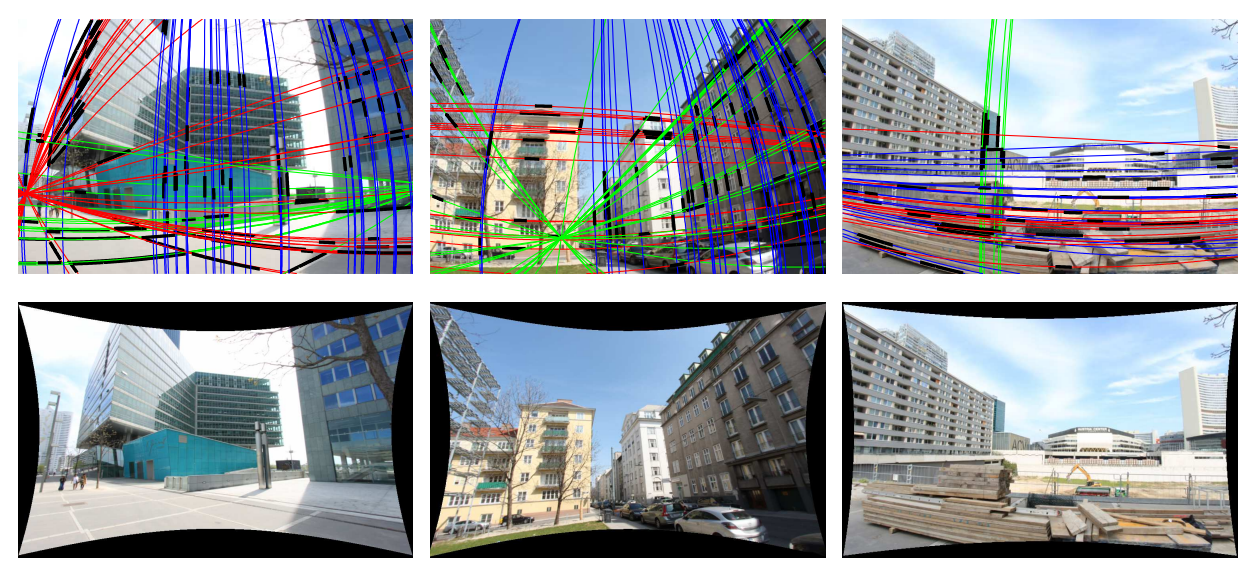

Figure 5: Exemplary results: Circular arcs (black) grouped w.r.t. three vanishing points, indicated by converging (red, green, blue) circles together with obtained undistorted images.

As one can see, the refinement gives a moderate improvement over the pure RANSAC method. R5CA+MLE obtained a relative error in radial distortion estimation of less than 5\% in more than $75 \%$ of trials. Unsurprisingly, in terms of focal length estimation R4L+MLE works slightly better than our approach: It operates on perfectly undistorted images and has one degree of freedom less to deal with, both during feature extraction and in the detection stage. However, compared to R4L+MLE's 92\% R5CA+MLE still attains $87 \%$ successful calibrations with a relative focal length error of less than $5 \%$.

Exemplary results of our algorithm are depicted in Fig. 5. The left columns show successful runs, while the rightmost column illustrates a typical failure mode. Due to the low resolution no arcs were extracted from the building face and the stack of wood distracted the computation of orthogonal vanishing points. Thus, radial distortion was severely underestimated and the focal length computation returned a bogus result.

In a second experimental run, we show that our algorithms can easily deal with data showing negligible radial distortion by tests on the well established York Urban Database (YUD) [6]. Results for focal length, together with a plot of the distribution of the estimated $\lambda$ values, are shown in Fig. 4, bottom row. R5CA+MLE is almost as good as R4L+MLE which is among the best performing algorithms on YUD. As one can see, the estimated $\lambda$ 's huddle tightly around to a peak at about $\lambda=-0.02$. Indeed, a visual inspection showed the presence of slight barrel distortion for which the estimated value seems to be reasonable.

Our methods were implemented in MATLAB, with R5CA and R5CA+MLE being done in $\mathrm{C}++$. On a Core $17 \mathrm{CPU}$, for an average of 350 arcs per image of our data set, R5CA and R5CA+MLE took $40 \mathrm{~ms}$ and $62 \mathrm{~ms}$ respectively.

\section{Conclusion}

This paper has extended the state-of-the-art in camera self-calibration from vanishing points by integrating estimation of radial distortion, focal length, and vanishing point positions in one algorithmic step. The main contribution is a closed-form solution for direct estimation of radial distortion and a vanishing point from three circular arcs, extracted from image edges. Based on this, we devised an efficient RANSAC approach, which, by use of two more arcs, 
is able to accurately estimate radial distortion, focal length, and three orthogonal vanishing points. Extensive experiments on real data have demonstrated the validity of the method.

An important insight is that (under mild assumptions) it is possible to directly estimate vanishing points in images with strong radial distortion with only little more effort than in the standard, undistorted case. Furthermore, our algorithm may be used to detect vanishing points in images unaffected by radial distortion without the danger of overfitting.

\section{References}

[1] O. Barinova, V. Lempitsky, E. Tretiak, and P. Kohli. Geometric image parsing in manmade environments. In $E C C V, 2010$.

[2] João P. Barreto and Kostas Daniilidis. Fundamental matrix for cameras with radial distortion. In $I C C V, 2005$.

[3] Christian Bräuer-Burchardt and Klaus Voss. A new algorithm to correct fish-eye- and strong wide-angle-lens-distortion from single images. In ICIP, 2001.

[4] Christian Bräuer-Burchardt and Klaus Voss. Automatic correction of weak radial lens distortion in single views of urban scenes using vanishing points. In ICIP, 2002.

[5] N. Chernov. Circular and Linear Regression: Fitting Circles and Lines by Least Squares. CRC Press/Taylor \& Francis, 2011.

[6] P. Denis, J. H. Elder, and F. J. Estrada. Efficient edge-based methods for estimating Manhattan frames in urban imagery. In ECCV, 2008.

[7] Frederic Devernay and Olivier D. Faugeras. Straight lines have to be straight. Mach. Vis. Appl., 13(1):14-24, 2001.

[8] Moumen T. El-Melegy and Aly A. Farag. Nonmetric lens distortion calibration: Closed-form solutions, robust estimation and model selection. In ICCV, 2003.

[9] Andrew W. Fitzgibbon. Simultaneous linear estimation of multiple view geometry and lens distortion. In $C V P R$, pages 125-132, 2001.

[10] Lazaros Grammatikopoulos, George Karras, and Elli Petsa. An automatic approach for camera calibration from vanishing points. ISPRS Journal of Photogrammetry and Remote Sensing, pages 64-76, 2007.

[11] Ciarán Hughes, Robert McFeely, Patrick Denny, Martin Glavin, and Edward Jones. Equidistant (f) fish-eye perspective with application in distortion centre estimation. IVC, 28(3):538-551, 2010.

[12] Zuzana Kukelova and Tomás Pajdla. A minimal solution to radial distortion autocalibration. IEEE T-Pami, (12):2410-2422, 2011.

[13] Branislav Micusik and Horst Wildenauer. Minimal solution for uncalibrated absolute pose problem with a known vanishing point. In International Conference on $3 D$ Vision (3DV), 2013. 
[14] B. Prescott and G. F. McLean. Line-based correction of radial lens distortion. CVGIP: Graphical Model and Image Processing, 59(1):39-47, 1997.

[15] Rickard Strand and Eric Hayman. Correcting radial distortion by circle fitting. In BMVC, 2005.

[16] SriRam Thirthala and Marc Pollefeys. The radial trifocal tensor: A tool for calibrating the radial distortion of wide-angle cameras. In CVPR, 2005.

[17] Horst Wildenauer and Allan Hanbury. Robust camera self-calibration from monocular images of manhattan worlds. In CVPR, 2012.

[18] Zhengyou Zhang. A flexible new technique for camera calibration. IEEE T-Pami, 22 (11):1330-1334, 2000. 